\title{
The Contour Extraction of Cup in Fundus Images for Glaucoma Detection
}

\author{
Anindita Septiarini, Hamdani, Dyna Marisa Khairina \\ Faculty of Computer Science and Information Technology, Mulawarman University, Samarinda, Indonesia
}

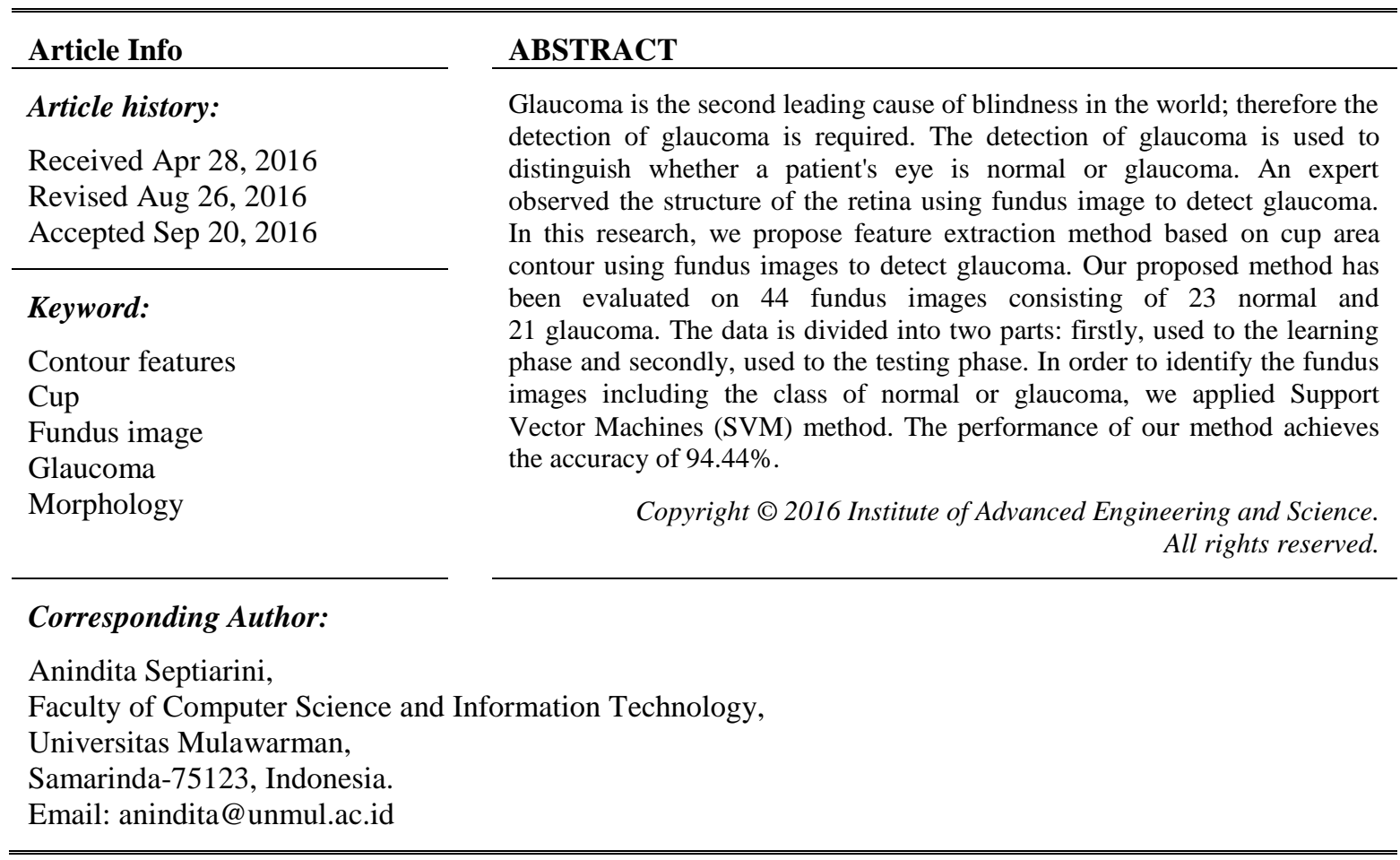

\section{INTRODUCTION}

Glaucoma is an eye disease that can cause vision problems. This disease is the second cause of blindness in the world [1], therefore the detection of glaucoma is required. The detection of glaucoma by an expert carried out by observing the structure of the retina using a fundus image, to determine the eyes of the patient are normal and glaucoma. Currently, research on automatic glaucoma detection techniques have been developed. Most of the research using fundus images as input data and several feature extraction methods have been applied.

Several previous researches related to automatic glaucoma detection generated by applying nonmorphological feature extraction method (image-based features). The feature referred to color, shape or texture on the optic nerve head $(\mathrm{ONH})$, retinal nerve fiber layer (RNFL) or blood vessel as part of the structure of the retina [2]. These parts undergo changes in patients with glaucoma [3]. ONH (also called disc) is the part area with round shape. Inside of the disc, there is a smaller area with round shape called a cup and a neuroretinal rim which is an area that lies between the disc and the cup. RNFL is the area outside of the disk where the structure of thickness of the RNFL is the one which distinguishes whether a patient's eye is normal or glaucoma. The illustration of ONH and RNFL structure are shown in Figure 1.

In the features extraction of the $\mathrm{ONH}$ applied the process of blood vessel inpainting as the previous phase in order to remove or blurring the blood vessel that presences as noise. This process is required the detection to determine the presence of the blood vessel. Several methods have been proposed for the detection of the blood vessel. Thresholding with the low pixel values in the image followed by median filtering of the size 41 x41 [4], using threshold probing [5] and normalized mathematical morphology (used to enhance the vessels) followed by neuro fuzzy classifier [6]. 
Subsequently, the feature extraction of the ONH based on shape proposed by using moment method in the image of Confocal Scanning Laser Tomography (CSLT) [7], Self-Organizing Map (SOM) [8] and Variational Expectation Maximization (VEM) on the image of Heidelberg Retina Tomograph (HRT) [9]. Several feature extraction methods of the texture approached by using Higher Order Spectra (HOS) method [10], the combination of HOS and Discrete Wavelet Transform (DWT) [11-12], wavelet sub-bands (wavelet subbands Daubechies (Db4), Symlets (sym4) and filter Biorthogonal (bi03.7, bi04.2 \& bi04.7)) [13]. Fractal Dimension [14], Gray Level Co-occurrence Matrix (GLCM) [15]. In 2007 Bock et al. has been implemented feature extraction by combining the shape, texture and color features using intensity value of pixel, Gabor Filter, coefficient, Fourier Transform (FFT) and model histogram method [16], then in 2010 he modified his research by different method: intensity of line pixel, FFT and B-spline [17].

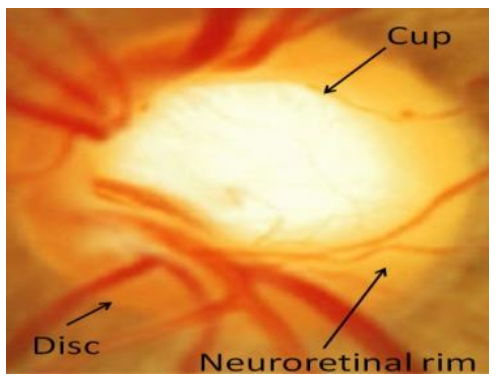

(a)

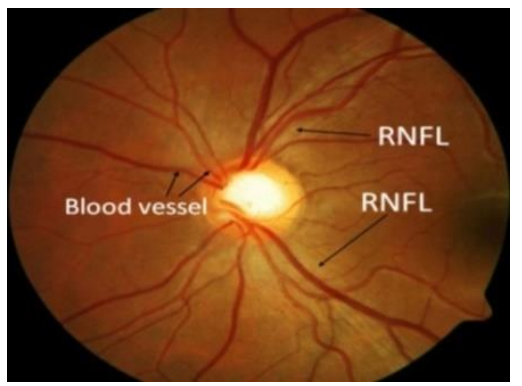

(b)

Figure 1. Structure of (a) ONH and (b) RNFL

In order to extract the feature of the RNFL, the previous researches applied Gaussian Markov random fields and local binary pattern method [18], fractal dimension [19-20]. Kolar and Jan (2008) used simple box counting, maximum likelihood estimators and spectral-based method [19], while Kim et al. (2013) used box-counting and multifractional Brownian motion [20]. The feature extraction towards blood vessel has done using vessel central axis detection, making the automatic module registration using retina images by self-organizing maps (SOMs) to look for some attributes such as connecting point, the form of the vessel [21].

The result of feature extraction produced data with large dimension, therefore to overcome the case, dimension reduction is required by Principal Component Analysis (PCA) [12], [16-17] or Sequential Forward Floating Selection (SFFS) to execute feature selection [15]. Furthermore, there are several methods used to the classification phase such as: Support Vector Machines (SVM) [7], [11], [16-17], Naïve Bayesian [10], statistical analysis [14], Multilayer Perceptron (MLP) [7], [21], Probabilistic Neural Network (PNN) [12], Learning Vector Quantization (LVQ) [8] and the combination of MLP with back propagation [13].

In this research, we propose the feature extraction of the ONH based on the cup area contour by simple methods of thresholding and morphology for glaucoma detection using fundus images. The use of fundus images due to several reasons such as the fundus camera widely found in health care center, cheaper, and it can be used to observe another disease: diabetic retinopathy and hypertensive retinopathy. The rest section in this paper we explain the research method in Section 2. The result and discussion are presented in Section 3. Finally, Section 4 concludes the paper.

\section{RESEARCH METHOD}

Our method divided into two main phase: the phase of learning and testing as illustrated in Figure 2. The initial input of each phase is the ONH image. It similar to the setup of the work in [22-23]. In the learning phase, data class (normal/glaucoma) is required as the input to give a label against the ONH image to determine whether the image is normal or glaucoma. The main phases of this research contain several processes, namely pre-processing, extracting features of the cup area and contour descriptor. The phase of learning and testing are terminated by the process saving the contour features and classification respectively.

\subsection{Pre-Processing}

The first step of this process was converting the ONH image (Figure 1(a)) as the initial input into 
green color because the area of the cup and the area of non-cup is more clearly distinguishable in this color [23-24]. Subsequently, we applied the process of blood vessel inpainting by the morphology closing operation followed by converting this result into grayscale image.

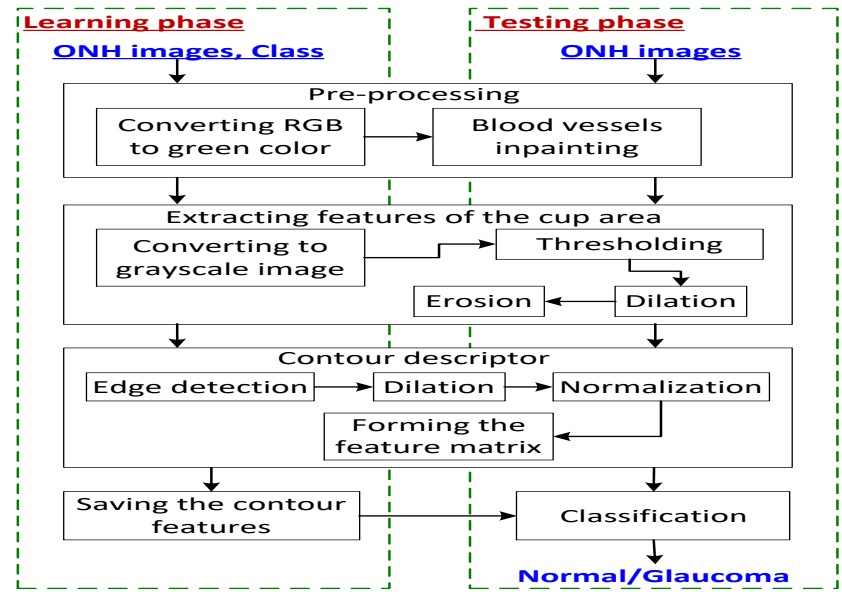

Figure 2. Block Diagram of our Proposed Method for Glaucoma Detection

\subsection{Extracting Features of the Cup Area}

The grayscale image in Figure 3(a) as the input of this process showed that the area of the cup has the higher intensity values $(I)$ than the surrounding area. In order to distinguish the area of the cup with the surrounding, we applied thresholding with the initial threshold value $(T)$ is the mean value of $I$ (see Figure 3(b) as the result). If the results of pixels thresholding reach the edge of the image, then update the $T$ value by increasing the $T$ value until none of the pixels thresholding reaches the edge of the image as show in Figure 3(c). Furthermore, the operation of erosion and dilation are applied to remove the pixels that predicted as the area of non-cup (Figure 3(d) as the result).

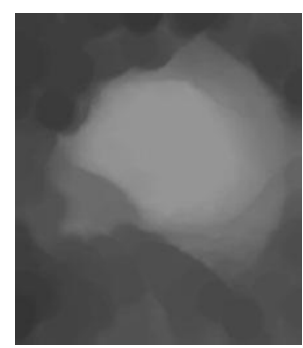

(a)

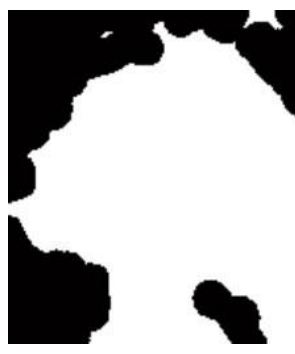

(b)

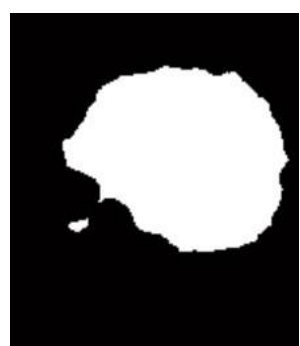

(c)

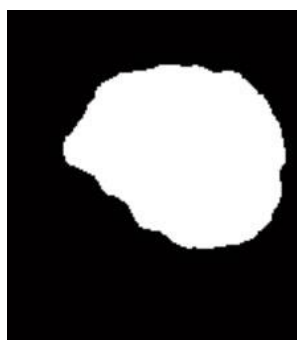

(d)

Figure 3. The Result of Extracting Features (a) Grayscale Image, (b) First Thresholding,

(c) Final Thresholding and (d) Morphology Operation

\subsection{Contour Descriptor}

The aim of the contour descriptor is to obtain the feature matrix based on the contour of the cup. The first step of this process is detection the edge of the cup using Sobel method, where the results are shown in Figure 4(a). Second, in order to combined the unconnected pixels in Figure 4(a) we applied dilation operation (see Figure 4(b)). Since the size of Figure 4(b) can be different, the normalizing process is required to formed sub-image with size $480 \times 480$ size and placed the contour of the cup in the middle of the image as shown in Figure 4(c). This image used as a reference to obtain the 2D feature matrix, by resizing Figure 3(c) into $240 \times 240$ pixels. The value of the feature matrix consists 0 or 1 based on the cup contour. 
Furthermore, in order to simplify the subsequential process, we reduced the size of the feature matrix by adding the content of feature matrix based on the square kernel (size 30x30) to a size $8 \times 8$. Figure 5 shows the illustration of the NxN feature matrix and the result of resized feature matrix based on the kernel with size $4 \times 4$.

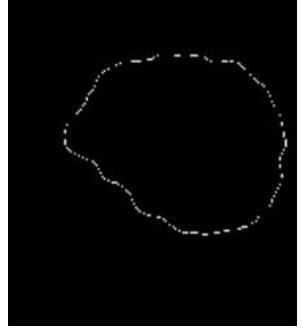

(a)

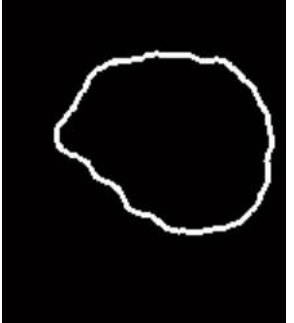

(b)

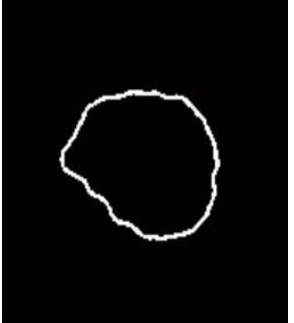

(c)

Figure 4. The Image Result of (a) Sobel Method, (b) Dilation Operation dan (c) Normalized Image

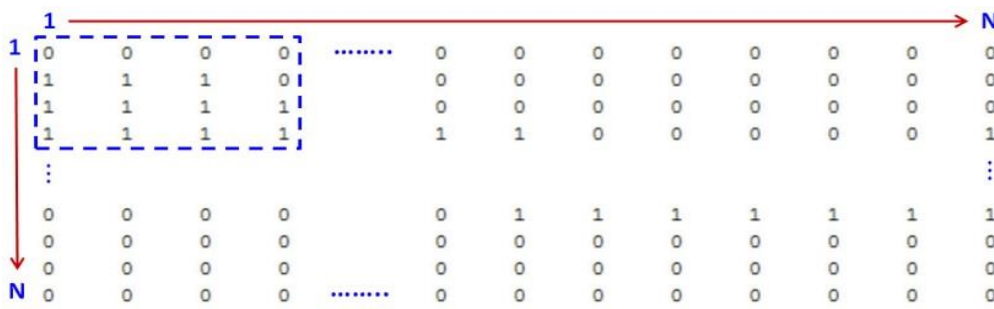

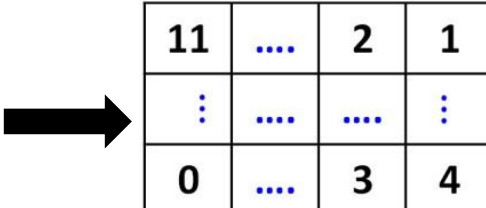

Figure 5. The Illustration of Reduction Process from the Feature Matrix

\subsection{Classification}

The final step of the testing phase, we used the feature matrix (size $8 \times 8$ ) as the input and applied the classification process to determine the $\mathrm{ONH}$ image was classified as normal or glaucoma class. In order to obtain a good classification result, determination of the hyperplane plays an important role. In this research, to produce the suitable hyperplane we applied SVM method.

\section{RESULTS AND DISCUSSION}

In this research, a dataset of the fundus images collected from Dr. YAP Eye Hospital in Yogyakarta that composed of $44 \mathrm{ONH}$ images (23 normal and 21 glaucoma) obtained from 44 full fundus images. The dataset consist of the fundus images of $\mathrm{ONH}$ with different size since the eye of patients have the ONH with varying sized. The fundus images captured by fundus camera Carl Zeiss AG with 30o field-of-view (FOV) and digital camera Nikon N150 of size 2240x1488 pixel. A total of 27 images (13 normal and 13 glaucoma) and 18 images (10 normal and 8 glaucoma) used as experimental data in the phase of learning and testing respectively. For each image was labeled as class of normal or glaucoma by a glaucoma expert. The examples of the ONH images with different size (in pixels) and class label as shown in Figure 6.

We used the value of precision, recall, Fscore and accuracy to measure the performance of our proposed method. The value of precision, recall and Fscore lies between 0 and 1, while the value of accuracy lies between 1 and 100. In order to indicates an accurate method they should have high values. The value of precision, recall, Fscore and accuracy are defined as follows:

$$
\begin{aligned}
& \text { precision }=\frac{T P}{T P+F P} \\
& \text { recall }=\frac{T P}{T P+F N}
\end{aligned}
$$




$$
\begin{aligned}
& F_{\text {score }}=2 \frac{\text { precision } \mathrm{X} \text { recall }}{\text { precision }+ \text { recall }} \\
& \text { Accuracy }=\frac{T P+T N}{T P+F P+F N+T N}
\end{aligned}
$$

where

True Positive (TP): is the number of class normal classified as class normal True Negative (TN): is the number of class glaucoma classified as class glaucoma False Positive (FP): is the number of class glaucoma classified as class normal False Negative (FN): is the number of class normal classified as class glaucoma
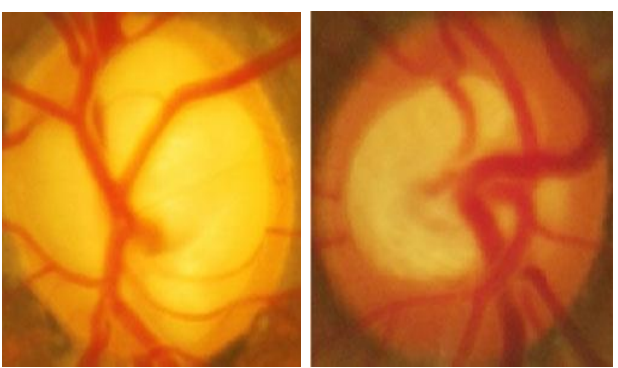

Size : 394 X 393

Class : Glaucoma

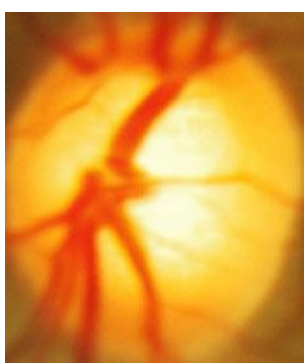

Size : 392 X 416

Class : Glaucoma

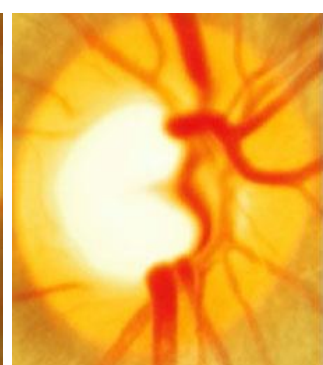

Size 375 X 423

Class : Normal

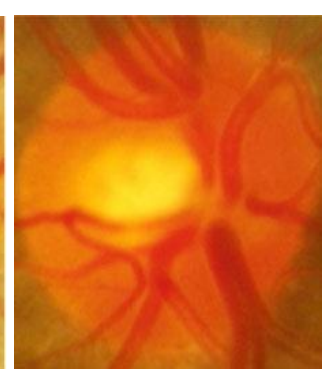

Size 311 X 345

Class : Normal

Figure 6. The ONH Images with Different Size (in pixels) and Class Label

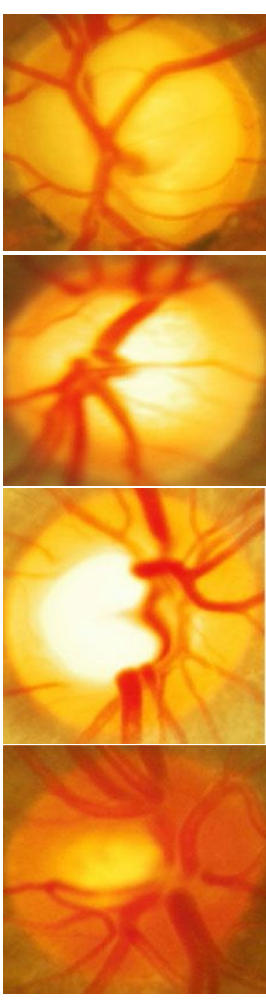

(a)

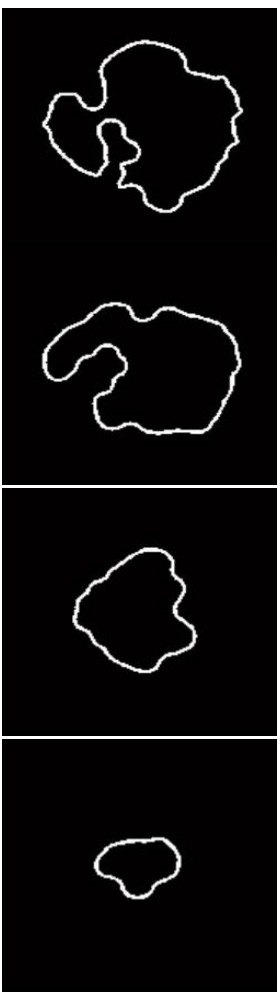

(b)

\begin{tabular}{|lrrrrrrr|}
\hline 0 & 0 & 0 & 0 & 0 & 0 & 0 & 0 \\
0 & 0 & 44 & 110 & 118 & 11 & 0 & 0 \\
0 & 12 & 108 & 136 & 73 & 139 & 0 & 0 \\
0 & 120 & 27 & 77 & 144 & 211 & 3 & 0 \\
0 & 107 & 0 & 0 & 8 & 3 & 140 & 0 \\
0 & 64 & 81 & 0 & 0 & 37 & 96 & 0 \\
0 & 0 & 116 & 114 & 118 & 87 & 0 & 0 \\
0 & 0 & 0 & 0 & 0 & 0 & 0 & 0 \\
\hline \hline 0 & 0 & 0 & 0 & 0 & 0 & 0 & 0 \\
0 & 0 & 40 & 114 & 99 & 0 & 0 & 0 \\
0 & 4 & 117 & 109 & 57 & 108 & 2 & 0 \\
0 & 59 & 69 & 101 & 108 & 63 & 45 & 0 \\
0 & 0 & 114 & 0 & 0 & 3 & 107 & 0 \\
0 & 0 & 106 & 0 & 0 & 6 & 104 & 0 \\
0 & 0 & 80 & 108 & 108 & 102 & 1 & 0 \\
0 & 0 & 0 & 0 & 0 & 0 & 0 & 0 \\
\hline \hline 0 & 0 & 0 & 0 & 0 & 0 & 0 & 0 \\
0 & 0 & 0 & 0 & 0 & 0 & 0 & 0 \\
0 & 0 & 7 & 129 & 125 & 29 & 0 & 0 \\
0 & 0 & 125 & 1 & 0 & 123 & 0 & 0 \\
0 & 6 & 150 & 6 & 2 & 124 & 0 & 0 \\
0 & 0 & 30 & 101 & 122 & 63 & 0 & 0 \\
0 & 0 & 0 & 0 & 0 & 0 & 0 & 0 \\
0 & 0 & 0 & 0 & 0 & 0 & 0 & 0 \\
\hline 0 & 0 & 0 & 0 & 0 & 0 & 0 & 0 \\
0 & 0 & 0 & 0 & 0 & 0 & 0 & 0 \\
0 & 0 & 0 & 44 & 42 & 0 & 0 & 0 \\
0 & 0 & 0 & 113 & 117 & 14 & 0 & 0 \\
0 & 0 & 0 & 115 & 115 & 10 & 0 & 0 \\
0 & 0 & 0 & 57 & 36 & 0 & 0 & 0 \\
0 & 0 & 0 & 0 & 0 & 0 & 0 & 0 \\
0 & 0 & 0 & 0 & 0 & 0 & 0 & 0 \\
\hline & & & & & & & \\
\hline 0 & 0 & 0 & 0 \\
\hline
\end{tabular}

(c)

Figure 7. Features Matrix Formation Step, Collumn (a) Original Image, (b) Cup Area Contour and (c) Features Matrix of Cup Area Contour 
In order to determine the performance of our method, we compared the classification results of the $\mathrm{ONH}$ images of our method by forming the feature matrix against the classification results of the expert based on observation of the ONH image. Several processes were applied to the ONH image (Figure 7(a)) as the initial input to produce the contour of the cup area (Figure 7(b)) up to form the feature matrix as illustrated in Figure 7(c).

We evaluated our proposed method using four kinds of classification methods namely MLP, Naive Bayes, k-NN and SVM to find out the optimal classification method. Table 1 compares all the classification methods in terms of average precision, recall, Fscore and accuracy.

The performance of our method is summarized in Table 1. The table shows that our method achieves the high value of precision, recall and Fscore of 0.95, 0.94 and 0.94 respectively, while the accuracy of $94.44 \%$ using SVM method as classifier. There are a few insights from our experiment result related to glaucoma detection in term of the extraction features. In this research, the combined of the contour extraction of the cup and SVM method as classifier produce competitive result. It is based on the comparison of the results with several previous reaserces which use different feature extraction method as shown in Table 2.

Table 1. The Comparison Performance of Classification Method

\begin{tabular}{|c|c|c|c|c|c|}
\hline Classifier & Class & Precision & Recall & Fscore & Accuracy (\%) \\
\hline \multirow{3}{*}{ MLP } & Glaucoma & 0.64 & 0.87 & 0.74 & \multirow{3}{*}{72.22} \\
\hline & Normal & 0.86 & 0.6 & 0.71 & \\
\hline & Average & 0.76 & 0.72 & 0.72 & \\
\hline \multirow{3}{*}{ Naïve Bayes } & Glaucoma & 0.67 & 1.0 & 0.80 & \multirow{3}{*}{77.78} \\
\hline & Normal & 1.0 & 0.6 & 0.75 & \\
\hline & Average & 0.85 & 0.78 & 0.77 & \\
\hline \multirow{3}{*}{$\mathrm{k}-\mathrm{NN}$} & Glaucoma & 0.8 & 1.0 & 0.89 & \multirow{3}{*}{88.89} \\
\hline & Normal & 1.0 & 0.8 & 0.89 & \\
\hline & Average & 0.91 & 0.89 & 0.89 & \\
\hline \multirow{3}{*}{ SVM } & Glaucoma & 1.0 & 0.87 & 0.93 & \multirow{3}{*}{94.44} \\
\hline & Normal & 0.91 & 1.0 & 0.95 & \\
\hline & Average & 0.95 & 0.94 & 0.94 & \\
\hline
\end{tabular}

Table 2. The Comparison of Results with other Researchs

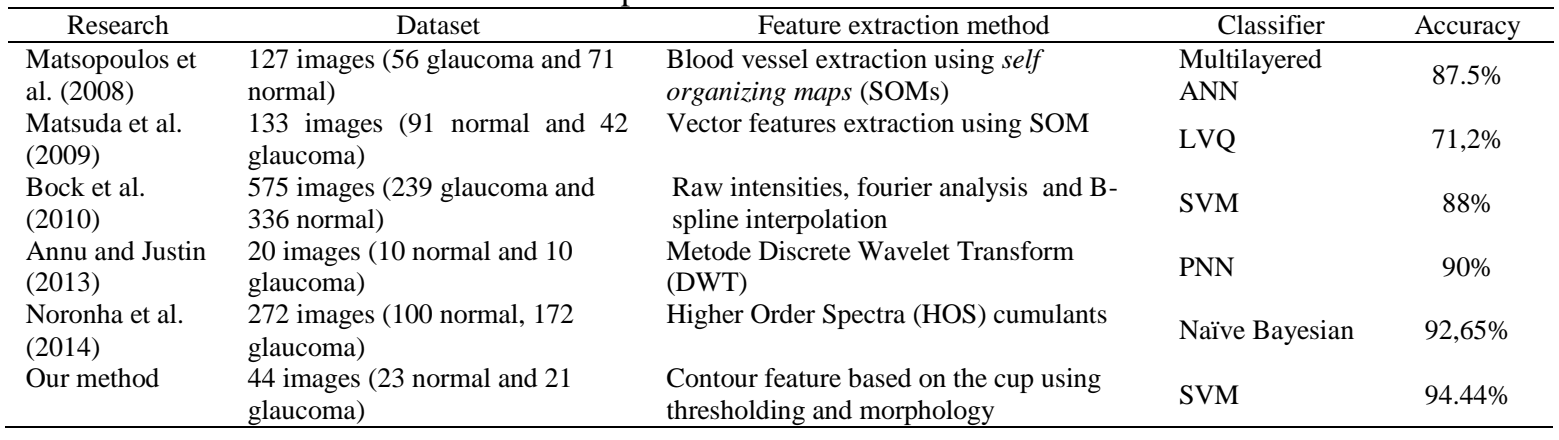

Based on table 2, glaucoma detection produced by several approaches, such as Blood vessel extraction using SOMs [21], Vector features extraction using SOM [8], Raw intensities, fourier analysis and B-spline interpolation [17], DWT method [12] and HOS cumulants [10]. We compare the performance of our proposed method based on the accuracy achievement. The accuracy achieved by [21] is $87.5 \%$, [8] is $71.2 \%$, [17] is $88 \%,[12]$ is $90 \%$ and [10] is $92.65 \%$.

\section{CONCLUSION}

In this research, we propose glaucoma detection method based on the contour extraction of the cup in fundus images. The proposed method evaluated against 44 images consisting of 23 normal and 21 glaucoma. It achieves the value of the accuracy of $94.44 \%$ using SVM method as the classifier. It proves that the contour of the cup is a reliable feature that can be used in the glaucoma detection 


\section{ACKNOWLEDGEMENTS}

This research was supported by Dr. YAP Eye Hospital Yogyakarta Indonesia for providing the experimental data to used in this research.

\section{REFERENCES}

[1] H.A. Quigley, "Number of People with Glaucoma Worldwide", Br. J. Ophthalmol., vol. 80, no. 5, pp. 389-93, May 1996.

[2] A. Septiarini and A. Harjoko, "Automatic Glaucoma Detection Based on The Type of Features Used: A Review", J. Theor. Appl. Inf. Technol., vol. 72, no. 3, pp. 366-375, 2015.

[3] G.K. Krieglstein and R.N. Weinreb, Glaucoma (Essentials in Ophthalmology). Springer-Verlag Berlin Heidelberg, 2009.

[4] H. Tjandrasa, A. Wijayanti, and N. Suciati, "Optic Nerve Head Segmentation Using Hough Transform and Active Contours", TELKOMNIKA, vol. 10, no. 3, pp. 531-536, 2012.

[5] M. Usman Akram, I. Jamal, and A. Tariq, "Blood Vessel Enhancement and Segmentation For Screening of Diabetic Retinopathy", Telkomnika, vol. 10, no. 2, pp. 327-334, 2012.

[6] R. Akhavan and K. Faez, "Two Novel Retinal Blood Vessel Segmentation Algorithms", Int. J. Electr. Comput. Eng., vol. 4, no. 3, pp. 398-410, 2014.

[7] J. Yu, S. Sibte, R. Abidi, P.H. Artes, and A. Mcintyre, "Automated Optic Nerve Analysis for Diagnostic Support in Glaucoma", in Proceedings of the 18th IEEE Symposium on Computer-Based Medical Systems, 2005.

[8] N. Matsuda, J. Laaksonen, F. Tajima, and H. Sato, "Classification of Fundus Images for Diagnosing Glaucoma by Self-Organizing Map and Learning Vector", in International Conference on Neural Information Processing, 2009, pp. 703-710.

[9] A. Belghith, M. Balasubramanian, C. Bowd, R.N. Weinreb, and L.M. Zangwill, "A Unified Framework for Glaucoma Progression Detection using Heidelberg Retina Tomograph Images”, Comput. Med. Imaging Graph., vol. 38, no. 5, pp. 411-420, 2014.

[10] K.P. Noronha, U.R. Acharya, K.P. Nayak, R. Joy, and S.V Bhandary, "Automated Classification of Glaucoma Stages using Higher Order Cumulant Features", Biomed. Signal Process. Control, vol. 10, pp. 174-183, 2014.

[11] M.R.K. Mookiah, U.R. Acharya, C.M. Lim, A. Petznick, and J.S. Suri, "Data Mining Technique for Automated Diagnosis of Glaucoma using Higher Order Spectra and Wavelet Energy Features", Knowledge-Based Syst., vol. 33, pp. 73-82, 2012.

[12] N. Annu and J. Justin, "Classification of Glaucoma Images using Wavelet based Energy Features and PCA", Int. J. Sci. Eng. Res., vol. 4, no. 5, pp. 1369-1374, 2013.

[13] R. Gayathri, P.V Rao, and S. Aruna, "Automated Glaucoma Detection System based on Wavelet Energy features and ANN", in Internationai Conference on Advances in Computing, Communications and Informatics, 2014, pp. $2808-2812$.

[14] D. Lamani, Ramegowda, and T. Manjunath, "Fractal Dimension as Diagnostic Parameter to Detect Glaucoma", Int. J. Innov. Eng. Technol., vol. 2, no. 1, pp. 63-69, 2013.

[15] S. Karthikeyan and N. Rengarajan, "Performance Analysis of Gray Level Co-Occurrence Matrix Texture Features for Glaucoma Diagnosis”, Am. J. Appl. Sci., vol. 11, no. 2, pp. 248-257, 2014.

[16] R. Bock, J. Meier, G. Michelson, L.G. Nyul, and J. Hornegger, "Classifying Glaucoma with Image-Based Features from Fundus Photographs", Springer-Verlag, pp. 355-364, 2007.

[17] R. Bock, J. Meier, L.G. Nyúl, J. Hornegger, and G. Michelson, "Glaucoma risk index : Automated Glaucoma Detection from Color Fundus Iimages”, Med. Image Anal., vol. 14, no. 3, pp. 471-481, 2010.

[18] J. Odstrcilik, R. Kolar, R. Tornow, J. Jan, A. Budai, M. Mayer, M. Vodakova, R. Laemmer, M. Lamos, Z. Kuna, J. Gazarek, T. Kubena, P. Cernosek, and M. Ronzhina, "Thickness related textural properties of retinal nerve fiber layer in color fundus images", Comput. Med. Imaging Graph., vol. 38, no. 6, pp. 508-516, 2014.

[19] R. Kolar and J. Jan, "Detection of Glaucomatous Eye via Color Fundus Images Using Fractal Dimensions", RADIOENGINEERING, vol. 17, no. 3, pp. 109-114, 2008.

[20] P.Y. Kim, K.M. Iftekharuddin, P.G. Davey, M. Toth, A. Garas, G. Hollo, and E.A. Essock, "Novel Fractal FeatureBased Multiclass Glaucoma Detection and Progression Prediction", IEEE J. Biomed. Heal. INFORMATICS, vol. 17, no. 2, pp. 269-276, 2013.

[21] G.K. Matsopoulos, P.A. Asvestas, K.K. Delibasis, N.A. Mouravliansky, and T.G. Zeyen, "Detection of Glaucomatous Change Based on Vessel Shape Analysis", Comput. Med. Imaging Graph., vol. 32, pp. 183-192, 2008.

[22] N. Tan, Y. Xu, W. B. Goh, and J. Liu, "Robust Multi-Scale Superpixel Classification For Optic Cup Localization", Comput. Med. Imaging Graph., vol. 40, pp. 182-193, Mar. 2015.

[23] H. Ahmad, A. Yamin, A. Shakeel, S.O. Gillani, and U. Ansari, "Detection of Glaucoma Using Retinal Fundus Images", pp. 321-324, 2014.

[24] J. Nayak and R.A.U, “Automated Diagnosis of Glaucoma Using Digital Fundus Images”, J. Med. Syst., vol. 33, pp. 337-346, 2009. 


\section{BIOGRAPHIES OF AUTHORS}
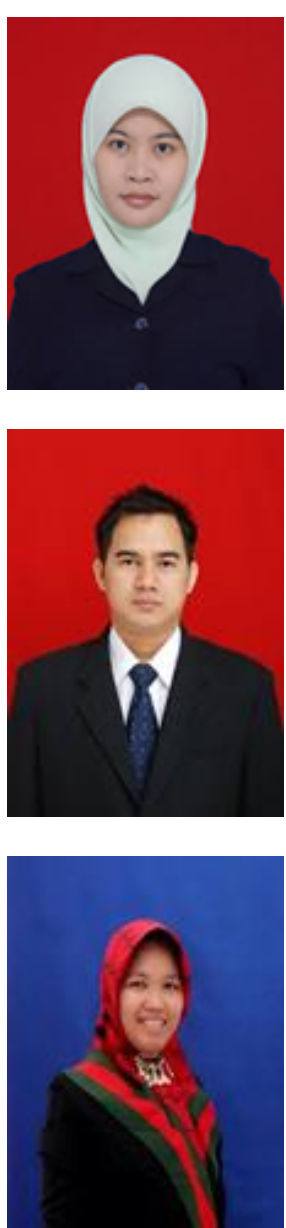

Anindita Septiarini was born in Nganjuk, East Java, Indonesia in September 1st 1982. She received her Bachelor degree in Informatic Engineering of Universitas Surabaya, Indonesia in 2005. She received her Master of Computer Scince degree at Universitas Gadjah Mada, Yogyakarta, Indonesia in 2009. Currently, she is attending doctoral program in Department of Computer Science, Universitas Gadjah Mada, Yogyakarta, Indonesia. She is working as a lecture in the department of Computer Science Universitas Mulawarman, Samarinda, Indonesia since 2009. Her research interest are image processing, pattern recognition and neural network.

Hamdani was born in Muara Bengkal, East Kalimantan, Indonesia in June 6th 1979. He received his Bachelor degree in Informatic Engineering of Universitas Ahmad Dahlan, Yogyakarta, Indonesia in 2002. He received her Master of Computer Scince degree at Universitas Gadjah Mada, Yogyakarta, Indonesia in 2009. Currently, he is attending doctoral program in Department of Computer Science, Universitas Gadjah Mada, Yogyakarta, Indonesia. $\mathrm{He}$ is working as a lecture in the department of Computer Science Universitas Mulawarman, Samarinda, Indonesia since 2005. His research interest are Group Decision Support, Data Security and Information System.

Dyna Marisa Khairina was born in Samarinda, East Kalimantan, Indonesia in Maret 5th 1984 She received her Bachelor degree in Computer Science of Universitas Mulawarman, Indonesia in 2007. She received her Master of Information System degree at Universitas Diponegoro, Semarang, Indonesia in 2012. She is working as a lecture in the department of Computer Science Universitas Mulawarman, Samarinda, Indonesia since 2008. Her research interest are information system and decision support system. She is meember of IAAII (Ikatan Ahli Informatika Indonesia) and APTIKOM (Association of Computing and Informatics Institution Indonesia) 